\title{
Vivre avec un cancer et d'autres maladies chroniques : perceptions des patients au sujet de leur expérience des soins de santé
}

\section{par Kia Duthie, Fay J. Strohschein et Carmen G. Loiselle \\ RÉSUMÉ}

On sait que la multimorbidité contribue à la complexité des soins prodigués aux patients atteints de cancer. La présente étude qualitative explore le vécu des patients qui reçoivent des traitements multimodaux (traitements pour de multiples maladies chroniques), ainsi que les difficultés pour ces patients à naviguer dans le système de soins de santé. Les participants $(n=10)$ ont été recrutés dans un centre ambulatoire de cancérologie d'un grand hôpital affilié à une université, à Montréal (province de Québec). Ils ont rapporté qu'il était particulièrement difficile d'atteindre un juste équilibre entre les besoins de santé aigus et la présence de maladies chroniques persistantes, de vivre des complications imprévues liées au traitement, et d'arriver à pallier le manque de liaison entre les spécialités médicales. Les participants auraient également souhaité que l'équipe soignante les connaisse mieux. Lorsqu'ils signalaient une expérience de coordination des soins positive, les participants l'associaient souvent à l'intervention d'une infirmière pivot. Finalement, les participants auraient aimé que l'approche de soins soit plus personnalisée et qu'ils puissent se joindre aux réunions du comité multidisciplinaire. Les résultats de l'étude soulignent le désir d'améliorer l'intégration des soins entre les maladies, les soins axés sur la personne et le soutien des patients qui cherchent à équilibrer des besoins concurrents en contexte de multimorbidité.

\section{INTRODUCTION}

T e nombre d'adultes diagnostiqués d'un cancer égale$ـ$ ment atteints d'une ou plusieurs maladies chroniques est en hausse constante. Le vieillissement s'accompagne d'une

\section{AU SUJET DES AUTEURES}

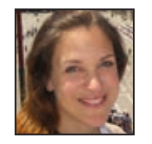

Kia Duthie, inf. aut., M.Sc., Infirmière de chevet, BC Cancer Agency, 401 - 122 Walter Hardwick Avenue, Vancouver, C.-B., Canada V5Y 0C9

\section{4-726-3902; kia.duthie@gmail.com}

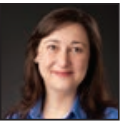

Fay J. Strohschein, inf. aut., Ph.D. (candidate), École des sciences infirmières Ingram, Université McGill, Conseillère en soins infirmiers, Centre du cancer Segal, Hôpital général juif, 3755, chemin de la Côte-Sainte-Catherine, Pavillon H-304.4, Montréal, QC, Canada H3T 1E2

514-340-8222, poste 3864; fay.strohschein@mail.mcgill.ca

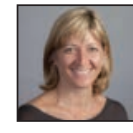

Auteure-ressource : Carmen G. Loiselle, inf., Ph.D., Professeure agrégée, Département d'oncologie, École des sciences infirmières Ingram, Université McGill, Codirectrice (recherche et enseignement) et chercheuse principale, Centre du cancer Segal, Hôpital général juif, 3755, chemin de la Côte-Sainte-Catherine, Pavillon E-748, Montréal, QC, Canada H3T 1E2

514-340-8222, poste 3940; carmen.loiselle1@mcgill.ca

DOI: $10.5737 / 236880762714955$ plus grande incidence du cancer et d'autres maladies chroniques comme le diabète, la maladie pulmonaire obstructive chronique, la cardiopathie, l'arthrite et l'hypertension (Société canadienne du cancer, 2012). Au moins 78 \% des personnes âgées (60 ans et plus) atteintes de cancer sont également atteintes d'une ou plusieurs maladies chroniques (Deckx et al., 2012; Ritchie et al., 2011). Les personnes âgées atteintes de trois maladies chroniques ou plus vont chez le médecin près de trois fois plus souvent que les personnes sans maladie chronique (Institut canadien d'information sur la santé, 2011). Comme le nombre de personnes souffrant du cancer en même temps que de maladies cooccurrentes s'accroît (Comité consultatif de la Société canadienne du cancer, 2015), une meilleure compréhension du vécu dans le domaine des soins de santé est nécessaire pour cette population aux besoins complexes.

\section{Multimorbidité et expérience du cancer}

On définit la multimorbidité comme la coexistence de deux ou plusieurs maladies chroniques ou aiguës chez une même personne (Marengoni et al. 2011; van den Akker et al., 1996). La multimorbidité peut influencer considérablement le vécu des patients aux prises avec une maladie chronique telle que le cancer de même que leurs interactions avec le système de soins de santé (Smith et al., 2008; Geraci et al., 2005). Plusieurs études démontrent que la cooccurrence de maladies affecte souvent négativement toutes les étapes de la trajectoire de soins du cancer, y compris le diagnostic, le pronostic, le traitement et la survie (Extermann, 2007; Louwman et al., 2005; Meyerhardt et al., 2003). Cette cooccurrence peut également aggraver la réponse du patient au traitement, le pronostic, le taux de survie et les effets subséquents sur la santé (Extermann, 2007; Meyerhardt et al., 2003; Wolf et al., 2005). Les personnes âgées atteintes de cancer sont moins susceptibles de recevoir un traitement agressif à visée curative (Partenariat canadien contre le cancer, 2016a), ce qui peut être dû, en partie, à la cooccurrence d'autres maladies chroniques (Chen et al., 2012; Srokowski et al., 2009).

\section{Multimorbidité et planification du traitement}

La planification du traitement constitue une source de préoccupations lorsqu'elle porte sur des interactions complexes entre le cancer et les maladies cooccurrentes. Lorsqu'une personne atteinte de maladies multiples reçoit un diagnostic de cancer, les décisions en matière de traitement doivent intégrer, coordonner et prioriser les modalités de traitements (Boyd et al., 2005; Yancik et al., 1998). Par exemple, l'équipe soignante doit se montrer vigilante quant aux interactions médicamenteuses possibles pendant le processus de traitement, car les lignes directrices actuelles en matière de pratique 


\begin{tabular}{|l|l|l|l|}
\hline \multicolumn{4}{|l|}{ Tableau 1: Caractéristiques des participants } \\
\hline Âge & Sexe & Types de cancers & Maladies chroniques \\
\hline 62 & F & Cancer du sein, cancer colorectal & Myasthénie grave auto-immune \\
\hline 55 & F & Cancer de l'endomètre & Diabète \\
\hline 68 & M & Lymphome B & Diabète, hypertension, hypercholestérolémie, hémochromatose (nécessitant Coumadin) \\
\hline 62 & M & Cancer de la prostate & Dépression chronique \\
\hline 67 & M & Cancer colorectal & Hypertension, diabète, stimulateur cardiaque (nécessitant Coumadin) \\
\hline 52 & F & Cancer colorectal & Diabète, hypercholestérolémie \\
\hline 71 & F & Cancer du sein & Neuropathie périphérique, ostéoarthrite, épithélioma basocellulaire \\
\hline 79 & M & Myélome multiple & Glaucome, hypertension, hypercholestérolémie \\
\hline 70 & M & Leucémie lymphoïde chronique & Hyperthyrö̈die, hypertension, hypercholestérolémie \\
\hline 73 & F & Lymphome nodulaire et lymphome B & Lupus cutané, syndrome de Sjögren \\
\hline
\end{tabular}

clinique ne portent que sur une maladie à la fois (Lee et al., 2011; Lindenfeld et Kelly, 2010; Tinetti et al., 2004). Échouer à répondre aux besoins complexes des patients peut mener à des résultats indésirables (Boyd et al., 2005). Vu le nombre limité de ressources et de normes pour équipes soignantes, on voit augmenter le risque de complications imprévues liées au traitement et de réduction de la qualité de vie et de soins pour ces patients.

\section{Multimorbidité et défis posés par la coordination des soins}

Intégrer des plans de traitement du cancer aux soins primaires prodigués dans le cas de maladies chroniques pose divers défis : participation de multiples cliniciens, interactions médicamenteuses possibles, effets secondaires, besoins concurrents de soins de santé. Une gestion de santé optimale nécessite une communication fréquente entre les cliniciens, le patient et ses proches. Malheureusement, bien souvent la coordination des services et des communications n'est pas efficace, si bien que les besoins du patient ne sont pas comblés et que les résultats cliniques ne sont pas au rendez-vous (Burgers et al., 2010; Jabaaij et al., 2012). Les études indiquent que les personnes cumulant un nombre élevé de maladies chroniques rapportent une moins bonne expérience avec les fournisseurs de soins de santé (Bowker et al., 2006; Burgers et al., 2010).

Plusieurs études se sont penchées sur la perception, par les patients atteints du cancer, des soins et services reçus (Sitzia et Wood, 1998; Van Der, 1999; Saultz et Albedaiwi, 2004; Puts et al., 2012), mais peu ont exploré à fond les besoins des patients atteints de cancer et de maladies cooccurrentes. Cette étude qualitative a été conçue de manière à obtenir des descriptions détaillées du point de vue des patients (Magilvy et Thomas, 2009) quant à leur expérience avec les traitements multimodaux, les besoins souvent complexes en soins de santé, et la navigation dans le système. Tout en cherchant à maintenir une approche exploratoire qui permette l'émergence des préoccupations et perspectives des participants, cette étude a fondé son cadre conceptuel sur la littérature susmentionnée montrant le caractère complexe de la multimorbidité et sur l'importance accordée aux soins axés sur la personne (Kitson, et al., 2013; Lusk et Fater, 2013). En faisant ressortir les thèmes fréquents associés à la multimorbidité, les résultats de l'étude pourront contribuer à la pratique clinique actuelle et donner des pistes quant à la manière dont les équipes soignantes pourraient mieux répondre aux besoins complexes de cette population particulière de patients.

\section{MÉTHODES}

\section{Participants}

On a approché, dans un centre ambulatoire de cancérologie d'un grand hôpital universitaire de Montréal, au Québec, les patients diagnostiqués du cancer avec cooccurrence d'au moins une maladie chronique. Le centre offre des soins intégrés aux personnes atteintes de cancer : dépistage, diagnostic, traitement, gestion de la douleur et des symptômes, soins palliatifs, soutien psychosocial, etc. Les infirmières pivots des services d’oncologie ont recruté les participants qui réunissaient les critères suivants : 50 ans et plus, anglophone, diagnostic de cancer avec cooccurrence d'au moins une autre maladie, ayant reçu un traitement contre le cancer (chimiothérapie, radiothérapie et/ou intervention chirurgicale) dans les 12 derniers mois. La présence préalable de tout problème physique ou mental grave qui risquerait d'affecter la capacité à participer à l'étude menait à l'exclusion. Parmi les quatorze patients abordés, dix ont accepté de participer à l'étude (les quatre refus s'expliquant par un manque de temps). Les participants recrutés ( 5 femmes et 5 hommes) étaient âgés de 52 à 79 ans $(\mu=66)$ et ont déclaré volontairement leur diagnostic de cancer : cancer colorectal, du sein, lymphome à cellules B, cancer de l'endomètre, de la prostate, myélome multiple ou leucémie lymphoïde chronique, selon le cas (voir tableau 1). 


\section{Protocole d'entrevue}

Les participants ont été interrogés individuellement pendant 40 à 80 minutes soit à l'hôpital, en un lieu privé, soit à leur domicile. L'entrevue, semi-structurée, comportait notamment des questions ouvertes comme : «Comment se sont passés pour vous les traitements contre le cancer? » « Pouvezvous me parler des autres maladies ou problèmes de santé qui vous ont été diagnostiqués? » ou encore « Comment vous soignez-vous pour ces problèmes et maladies? » Les renseignements sociodémographiques ont quant à eux été recueillis à l'aide d'un questionnaire volontaire.

Les entrevues ont été enregistrées sous format numérique, ont été transcrites textuellement, puis ont fait l'objet d'une analyse thématique appliquée, une approche exploratoire axée sur la description et la compréhension des sentiments, des pensées et des comportements exprimés par des sujets dans un contexte spécifique (Guest et al., 2011). L'analyse intègre des techniques méthodologiques clés pour offrir « un ensemble de procédures rigoureuses, mais inductives, conçues pour cerner et examiner des thèmes tirés de données textuelles » (Guest et al., 2011, p. 15, traduction). Le cœur de cette approche réside dans le codage ouvert des transcriptions pour réduire les données à de courtes phrases ou descriptions en vue de faire ressortir les concepts clés. Les descriptions ou phrases connexes sont ensuite regroupées en catégories, étudiées pour la récurrence des concepts et des sens similaires, puis regroupées sous des thèmes clés (Magilvy et Thomas, 2009; Guest et al., 2011). Pour assurer la fiabilité des données (Lincoln et Guba, 1985; Polit et Beck, 2010; Tuckett, 2005; Koch, 2006), l'auteure principale a discuté pendant la collecte et l'analyse des données de son analyse première avec des collègues et des superviseurs universitaires, puis intégré le fruit de ces premières analyses dans les entrevues subséquentes pour vérifier les concepts analytiques avec les participants, en gardant des traces vérifiables en la totalité des enregistrements vocaux, des transcriptions et des notes détaillées liées au processus de recherche (y compris les étapes de collecte et d'analyse des données).

\section{RÉSULTATS}

L'analyse des données a révélé plusieurs thèmes clés. Le plus fondamental était lié au besoin des participants d'atteindre un «juste équilibre » entre les exigences liées au traitement de leur cancer et les exigences liées aux autres maladies chroniques dont ils souffraient. Les participants qui avaient le mieux réussi à atteindre cet équilibre ont expliqué vivre la situation comme une « approche holistique, personnalisée et bienveillante ». Du côté des expériences négatives, les participants ont exprimé des inquiétudes, ayant « l'impression que l'équipe soignante les connaissait mal ou ne les écoutait pas ». Les participants des deux groupes ont aussi spontanément identifié l'infirmière pivot (cette professionnelle de la santé qui accompagne et soutient les patients et leur famille tout au long de la trajectoire de soins) comme un élément positif et essentiel ayant contribué à la qualité des soins et les ayant aidés à gérer la multimorbidité. Un autre thème qui émergeait des besoins exprimés par les participants était le souhait d'être présents lors des réunions multidisciplinaires (aussi appelées « réunions d'études de cas » ou « rencontres du comité des tumeurs »).
Trouver un juste équilibre : défis concrets liés à la multimorbidité

Si l'on en croit l'analyse des données, la capacité des participants à gérer le traitement contre le cancer était plus difficile en présence de certaines maladies chroniques comme le diabète et les maladies nécessitant une médication comme le Coumadin. Les participants diabétiques, par exemple, ont exprimé des difficultés importantes, notamment, pour les soins autoadministrés, les interactions médicamenteuses, les effets secondaires douloureux ou inquiétants, les erreurs de soins ou de médication, les préoccupations financières et la mauvaise coordination des soins. Ces participants ont aussi rapporté des difficultés à gérer leur diabète en raison des traitements contre le cancer. Par exemple, un participant à qui on avait prescrit de la prednisone pour traiter son cancer s'est retrouvé avec des taux de glycémie élevés à gérer. Trouver le bon dosage de Coumadin a aussi fait partie des difficultés relevées en lien avec le traitement du cancer : «Tout va de travers pour le moment à cause de la chimiothérapie. » Un participant a ainsi fait état d'effets secondaires dus à l'augmentation des doses de Coumadin, comme le retard de coagulation et de guérison, et un autre participant a fait part des soucis financiers créés par un changement de médicament dû à l'incompatibilité du Coumadin avec son traitement contre le cancer, le nouveau médicament étant « extrêmement cher » et lui causant donc bien du stress.

Les participants atteints d'autres maladies chroniques comme le lupus cutané, l'hypertension, l'hypercholestérolémie, l'hyperthyroïdie, la dépression et l'ostéoarthrite n'ont pas trouvé que le traitement de leur cancer avait eu de répercussions importantes sur la gestion de leurs maladies chroniques. Ils ont décrit un équilibre relatif atteint à cet égard et leur permettant de se concentrer sur le traitement contre le cancer comme nouvelle priorité : «Je prends mes autres médicaments comme j'ai l'habitude de le faire. Je le fais sans trop y penser, car ce cancer prend toute la place. » Les participants ont également décrit, en général, des expériences positives avec le système de soins de santé, la plupart de leurs besoins ayant obtenu réponse. Voici trois témoignages à cet égard : « Les infirmières, la façon dont le système fonctionne ici, c'est excellent »; « Les soins et la coordination des traitements que je reçois sont incroyablement bons »; et « Je crois que je vais bien et qu'on prend très bien soin de moi ».

\section{Sentiment d'être mal connu ou écouté par l'équipe soignante}

Aux difficultés éprouvées par certains participants pour équilibrer les exigences multiples liées à la multimorbidité s'est ajouté, pour ceux dont les besoins en soins de santé étaient non comblés, des lacunes évidentes quant aux soins reçus, et ce, malgré leurs demandes de soutien supplémentaire. L'impression « de ne pas être connus » ou « de ne pas être écoutés » par l'équipe soignante a été mentionnée à maintes reprises. Ces participants ont eu le sentiment que leurs fournisseurs de soins de santé ne lisaient pas complètement leur dossier médical ou comprenaient mal leur situation globale sur le plan médical : «À certains moments, j'avais l'impression qu'ils ne possédaient pas tous les renseignements, 
que les nombreuses personnes qui prenaient connaissance de mon dossier ne le lisaient pas attentivement. » Un participant a rapporté devoir souvent répondre aux mêmes questions et fournir les mêmes renseignements médicaux « encore et encore », ce qui a causé de la frustration : « Le fichu de dossier est pourtant là, allez donc le lire et vérifier vous-même! »

Les participants ont indiqué souhaiter une communication et un engagement plus clairs concernant la planification du traitement et la reconnaissance de leurs besoins en matière de soins de santé. Un participant s'est dit surpris, mais reconnaissant, qu'un chirurgien en particulier ait pris le temps de lui expliquer en détail son plan de traitement : «C'est le seul en six ou sept ans qui a autant pris le temps de m'expliquer. » Ce participant a souligné à quel point il était important de prendre le temps d'apprendre à connaître les patients et de les rassurer : « Vous traitez des gens, et bien traiter des gens, ça prend du temps. » Il a eu l'impression que ce temps consacré à répondre aux préoccupations des patients, seules « quelques personnes précises réellement à l'écoute » le prenaient. Cela illustre l'importance d'améliorer la communication entre le fournisseur de soins et le patient.

En l'absence d'explications et de renseignements clairs, les participants se sentent anxieux, confus et stressés, principalement par manque de compréhension quant aux processus et à certaines décisions de traitement. Une participante a été informée qu'elle avait un cancer agressif et à croissance rapide, mais qu'elle devait attendre avant de commencer la chimiothérapie. «Comment puis-je attendre comme ça "pour commencer"? », s'est-elle demandé. Elle a également dit ne pas comprendre pourquoi elle n'avait toujours pas subi d'intervention chirurgicale : « C'est stressant et bouleversant de vivre ça. »

\section{Une approche bienveillante intégrée et personnalisée}

Les participants qui n'ont pas vécu de complications liées à la gestion de leurs maladies chroniques ont rapporté avoir bénéficié d'une « approche de soins holistiques bienveillante » de la part de l'équipe soignante à qui ils ont fait part de leurs préoccupations : " On m’a dit que si j'avais des problèmes, qu'ils soient d'ordre financier ou autres, d'en parler et qu'ils pourraient me donner de l'aide »; «Ils ne veulent pas que rien ne nous inquiète ». Ces participants ont également mentionné l'importance du temps mis par les fournisseurs de soins pour adapter les soins prodigués aux besoins individuels de chaque patient : « Il apprend à connaître ses patients et sait comment ne leur faire part que des renseignements qu'il leur faut. » On a également mentionné que les membres de l'équipe ont fait en sorte qu'ils se sentent vraiment en sécurité et à l'aise : « La première fois que je suis venu ici, jétais la personne la plus effrayée qui soit. Maintenant, je me sens tellement à l'aise, et c'est grâce à vous [l'équipe soignante] ».

\section{L'infirmière pivot : composante essentielle pour la qualité des soins}

Les participants ont nommé l'infirmière pivot en oncologie comme la plus importante ressource pour obtenir de l'information, avoir du soutien émotionnel et améliorer la continuité des soins pendant tout le processus complexe du traitement du cancer. Les participants ont souvent exprimé leur gratitude envers l'infirmière pivot : «C'est ça qui m’a sauvé. Peu importe le problème que j'ai, si j'ai une interrogation, si je suis à la maison et que je suis inquiet, ou peu importe, je n'ai qu'à l'appeler. Et elle me rappelle »; « Elle sait tout, elle est là pour tout. Je pense que je parle plus souvent avec elle qu'avec mon médecin. Je me sens plus à l'aise avec elle »; «Elle a beaucoup de réponses claires et adaptées à mon cas ». Ces citations soulignent le rôle essentiel joué par l'infirmière pivot. Les participants ont également décrit le rôle important joué par l'infirmière pour faciliter la communication entre les membres de l'équipe soignante par rapport aux soins : « Elle me sert de liaison »; « Elle intervient et s'assure que toutes les personnes qui me traitent se parlent »; « On a besoin de quelqu'un qui puisse parler à tout le monde ».

\section{Participation aux réunions multidisciplinaires}

Un des désirs les plus marqués exprimés par les participants était d'être invités aux rencontres réunissant toute l'équipe soignante-infirmières, oncologues, chirurgiens et parfois même ombudsman de l'hôpital-pour pouvoir leur faire part de leur vécu personnel et de leurs problèmes : « Voici la manière dont je réagis, voici mon problème, voici comment mon corps réagit, comment mon intellect réagit, voici mon état émotionnel. » Les participants ont aussi signifié leur désir d'augmenter leurs connaissances en santé en posant des questions à toute l'équipe soignante, afin de pouvoir mieux participer au processus de planification du traitement. Cette idée suscitait aussi le sentiment qu'il serait alors possible d'être mieux écouté et connu comme patient. Un participant a rapporté ne pas avoir été consulté dans le cadre d'une longue évaluation détaillée par l'équipe : « Je me connais mieux que personne [...] Je sais comment mon corps réagit à certaines choses. »Un autre expliquait sa frustration de ne pas avoir été invité à participer à son plan de traitement et aux décisions concernant ses soins : " C'est ma vie après tout! [...] Je me connais, je sais ce qui me convient le mieux. » Ceci renforce la nécessité que l'équipe soignante reconnaisse l'importance et la valeur de la participation des patients dans la prise de décisions et l'élaboration des plans de soins.

\section{DISCUSSION}

Explorer le vécu en matière de soins de santé des patients ayant un cancer en même temps que d'autres maladies chroniques a fait ressortir les aspects essentiels suivants : l'atteinte d'un juste équilibre entre les besoins liés aux maladies chroniques et ceux liés aux maladies aiguës, ainsi que la nécessité de se sentir connu et écouté par l'équipe soignante. Ce dernier problème est activement contré par l'impulsion donnée pour aller vers ses soins axés sur la personne (Partenariat canadien contre le cancer, 2016b). Cette approche de soins individualisés et holistiques est personnalisée en fonction des besoins, valeurs et perspectives de chaque personne (Kitson, et al., 2013; Lusk et Fater, 2013). Les participants ont décrit l'importance ou le désir que l'équipe adopte une approche axée sur leurs besoins personnels et leurs préférences. Malgré de récents efforts pour réorienter les soins du cancer de manière à s'éloigner de l'approche paternaliste traditionnelle pour se 
recentrer sur la personne, les études semblent indiquer que les soins axés sur la personne continuent d'être un défi, particulièrement chez les patients aux prises avec des maladies multiples (Bayliss et al., 2008; Fashoyin-Aje et al., 2012). Il est essentiel d'inclure systématiquement les patients dans la définition et la mise en œuvre de ce que signifie pour eux l'idée de recevoir des soins axés sur la personne, afin d'améliorer le soutien et la qualité de soins dans son ensemble.

D'autres études ont signalé que les participants aux prises avec des maladies multiples rapportaient des interactions problématiques avec l'équipe soignante, le sentiment d'être négligés (Noël et al., 2005), ainsi que des besoins en matière de santé non comblés, de même qu'un manque de soins médicaux intégrés dû aux contraintes de temps pendant les consultations avec les fournisseurs de soins (Clarke et al., 2013), ce qui vient corroborer nos résultats. Une autre étude a établi que la clarté des communications, les plans de soins personnalisés et la reconnaissance des besoins par les fournisseurs de soins de santé étaient des processus de soins fortement désirés par les patients aux prises avec des maladies multiples (Bayliss et al., 2008). Pour atteindre cet objectif, il faudrait que les fournisseurs de soins connaissent mieux les antécédents médicaux $\mathrm{du}$ patient, aient une bonne compréhension de son portrait médical global avant de le rencontrer, et soient au courant des renseignements que chaque patient préfère ou pas recevoir concernant son cancer (Lambert, Loiselle, et Macdonald, 2009).

De plus, la clarté des communications entre le professionnel et le patient quant aux décisions concernant le traitement, aux effets secondaires possibles et aux interactions médicamenteuses potentielles est essentielle. Les fournisseurs de soins pourraient aussi vérifier périodiquement auprès des patients s'il y a des lacunes en matière de coordination des soins afin de réduire le risque de besoins non comblés ou de complications (Extermann, 2007; Louwman et al., 2005; Meyerhardt et al., 2003). Des soins mal coordonnés peuvent entraîner la répétition de questions médicales auprès des patients et une négligence à tenir compte des prochaines étapes de soins; il semble donc important de reformuler certaines questions des soins de santé, puis de les valider ensuite avec les patients : «J'ai vu dans votre dossier que... Est-ce toujours le cas pour vous? » (Clausen et al., 2012) Ainsi, les professionnels de la santé rassurent le patient, lui montrant qu'ils connaissent son dossier, et l'invitent à s'exprimer sur ses préoccupations actuelles quant à sa santé.

Les participants ont également exprimé le désir de faire partie des discussions multidisciplinaires, ce qui réduirait les lacunes communicationnelles et comblerait le besoin d'être connus par l'équipe soignante, comme indiqué précédemment. Il y a peu d'écrits sur la participation des patients à ces réunions, mais l'importance de faire participer les patients à l'élaboration de leur plan de soins a été soulignée (Clausen, et al., 2012). De plus, les recherches effectuées sur l'idée que la famille se concerte au chevet du patient (et, plus récemment, que les infirmières en fassent autant) ont montré que la participation du patient à ces conversations venait améliorer la communication dans la famille et avec l'équipe soignante, un résultat qui pouvait être corrélé avec une amélioration de la qualité des soins évalués, une plus grande satisfaction du patient, et une diminution de l'anxiété (Delgado et al., 2009). Malgré les efforts faits pour favoriser la participation du patient dans la prestation des soins de santé, les études oublient souvent de se pencher sur les rôles que les patients voudraient jouer plus particulièrement (Thompson, 2007). Notre constat ici est qu'il y a une préférence claire du participant : celle qu'il voudrait participer davantage aux rencontres et réunions de l'équipe soignante.

Une stratégie prometteuse pour améliorer la communication et la coordination des soins serait de faire participer les infirmières pivots (aussi appelées « infirmières navigatrices ») à toutes les étapes des soins de santé reçus pour le cancer et de la multimorbidité. L'infirmière pivot accompagnerait les patients ayant des besoins complexes dans tout leur parcours de soins du cancer, en évaluant et en prenant en charge les besoins du patient et de la famille, en donnant information et renseignements, et en offrant soutien et aide pour la coordination des soins et des services (De Serres et Beauchesne, 2000; Horner et al., 2013). Les participants ont désigné l'infirmière pivot comme une ressource essentielle pour le soutien émotionnel, les besoins d'information et la continuité des soins pendant qu'ils naviguent dans les dédales du système de soins de santé. Les patients pour qui la gestion des maladies chroniques compliquait les choses ont particulièrement insisté sur le rôle positif que l'infirmière pivot, en facilitant la communication entre les fournisseurs de soins et en servant de point de repère fixe et stable tout au long du parcours, a joué pour eux.

Il est important de noter que les participants à cette étude ont été recrutés par les infirmières pivots; ils pouvaient donc déjà avoir avec elles des liens plus étroits et donc véhiculer une opinion plus positive à leur égard (caractéristiques de la demande). Toutefois, Bayliss et al. (2008) ont eux aussi trouvé que les patients souffrant de multiples maladies désiraient le « soutien d'un seul coordonnateur de soins qui pourrait les aider à prioriser les exigences concurrentes émanantes de leurs multiples maladies dans le cadre d'une relation continue » (p. 289, traduction). D’autres études ont montré que les infirmières pivots en oncologie aident les patients à prendre en charge leurs symptômes et les autres maladies, réduisent la détresse liée au cancer, et augmentent dans l'ensemble le taux de satisfaction et de qualité de vie du patient (Swanson et Koch, 2010; Jennings-Sanders et Anderson, 2003). Les infirmières pivots devraient également être encouragées à communiquer régulièrement avec le médecin généraliste du patient, qui joue souvent un rôle actif dans la gestion des autres maladies du patient pendant la durée du traitement contre le cancer (Klabunde et al., 2009). Les patients atteints de cancer ont $54 \%$ plus de consultations et $68 \%$ plus d'ordonnances de leur médecin généraliste dans l'année suivant leur diagnostic (Brandenbarg et al., 2014).

Le fardeau associé à un diagnostic de cancer est souvent important, les soins médicaux devenant de plus en plus complexes et impliquant souvent de nombreuses autres spécialités de soins de santé. Ces défis sont amplifiés lorsque les patients ont aussi d'autres maladies chroniques. Dans ce contexte, 
répondre aux besoins psychosociaux, physiques, d'information et de coordination revêt une très grande importance. Nos constats soulignent les aspects importants des soins de santé axés sur la personne selon les patients et tendent à indiquer que les patients ayant des maladies multiples ont des besoins complexes supplémentaires en matière de planification du traitement et de coordination des soins. Cela prouve aussi une fois de plus l'importante valeur du rôle de l'infirmière pivot en oncologie. Ce rôle devrait continuer d'être développé et soutenu pour offrir des soins optimaux aux cancéreux présentant

\section{RÉFÉRENCES}

Bayliss, E.A., Edwards, A.E., Steiner, J.F. et Main, D.S. (2008). Processes of care desired by elderly patients with multimorbidities. Family Practice, 25, 287-293.

Bowker, S.L., Pohar, S.L. et Johnson, J.A. (2006). A cross-sectional study of health-related quality of life deficits in individuals with comorbid diabetes and cancer. Health and Quality of Life Outcomes, 4,1 .

Boyd, C.M., Darer, J., Boult, C., Fried, L.P., Boult, L. et Wu, A.W. (2005). Clinical practice guidelines and quality of care for older patients with multiple comorbid diseases: Implications for pay for performance. JAMA, 294, 716-724.

Brandenbarg, D., Roorda, C., Groenhof, F., Havenga, K., Berger, M.Y., de Bock, G.H. et Berendsen, A.J. (2014). Increased primary health care use in the first year after colorectal cancer diagnosis. Scandinavian Journal of Primary Health Care, 32, 55-61.

Burgers, J.S., Voerman, G.E., Grol, R., Faber, M.J. et Schneider, E.C. (2010). Quality and coordination of care for Patients with multiple conditions: Results from an international survey of patient experience. Evaluation Q the Health Professions, 33, 343-364.

Chen, R.C., Royce, T.J., Extermann, M. et Reeve, B.B. (2012). Impact of age and comorbidity on treatment and outcomes in elderly cancer patients. Seminars in Radiation Oncology, 22, 265-71.

Clarke, L.H., Bennett, E.V. et Korotchenko, A. (2013). Negotiating vulnerabilities: How older adults with multiple chronic conditions interact with physicians. Canadian Journal on Aging/La Revue canadienne du vieillissement, 33, 1-12.

Clausen, C., Strohschein, F., Faremo, S., Bateman, D., Posel, N. et Fleiszer, D. (2012). Developing an interprofessional care plan for an older adult woman with breast cancer: From multiple voices to a shared vision. Clinical Journal of Oncology Nursing, 16(1), E18E25. doi:10.1188/12.CJON.E18-E25

De Serres, M. et Beauchesne, N. (2000). L'intervenant pivot en oncologie : un rôle d'évaluation, d'information et de soutien pour le mieux-être des personnes atteintes de cancer: document de réflexion, [Québec] : Conseil québécois de lutte contre le cancer.

Deckx, L., van den Akker, M., Metsemakers, J., Knottnerus, A., Schellevis, F. et Buntinx, F. (2012) Chronic diseases among older cancer survivors. Journal of Cancer Epidemiology, 2012, 1-7.

Delgado, E.M., Callahan, A., Paganelli, G., Reville, B., Parks, S.M. et Marik, P.E. (2009). Multidisciplinary family meetings in the ICU facilitate end-of-life decision making. American Journal of Hospice and Palliative Medicine, 26, 295-302.

Extermann, M. (2007). Interaction between comorbidity and cancer. Cancer Control, 14, 13-22.

Fashoyin-Aje, L.A., Martinez, K.A. et Dy, S.M. (2012). New patientcentered care standards from the Commission on Cancer: Opportunities and Challenges. The Journal of Supportive Oncology, 10, 107-111. des cas de soins complexes comme des comorbidités multiples. Finalement, les résultats indiquent que les patients souhaitent participer aux réunions multidisciplinaires pour favoriser un consensus partagé dans les cas complexes, ou lorsque les priorités et les objectifs concernant le plan de soins diffèrent. Offrir une approche personnalisée et complète aux patients souffrant de maladies complexes est nécessaire pour optimiser les répercussions sur la santé et améliorer l'expérience du patient en matière de soins de santé.

Geraci, J.M., Escalante, C.P., Freeman, J.L. et Goodwin, J.S. (2005). Comorbid disease and cancer: The need for more relevant conceptual models in health services research. Journal of Clinical Oncology, 23, 7399-7404.

Guest, G., Macqueen, K.M. et Namey, E.E. (2011). Applied thematic analysis, Sage.

Horner, K., Ludman, E.J., Mccorkle, R., Canfield, E., Flaherty, L., Min, J., ..., Wagner, E.H. (2013). An oncology nurse navigator program designed to eliminate gaps in early cancer care. Clinical Journal of Oncology Nursing, 17, 43-48.

Institut canadien d'information sur la santé (2011). Les personnes âgées et le système de santé : quelles sont les répercussions des multiples affections chroniques? Consulté sur https://secure.cihi.ca/ free_products/air-chronic_disease_aib_fr.pdf

Jabaaij, L., Van Den Akker, M. et Schellevis, F. (2012). Excess of health care use in general practice and of comorbid chronic conditions in cancer patients compared to controls. BMC Family Practice, 13, 60.

Jennings-Sanders, A. et Anderson, E.T. (2003). Older women with breast cancer: Perceptions of the effectiveness of nurse case managers. Nursing Outlook, 51, 108-114.

Kitson, A., Marshall, A., Bassett, K. et Zeitz, K. (2013). What are the core elements of patient-centred care? A narrative review and synthesis of the literature from health policy, medicine and nursing. Journal of Advanced Nursing, 69(1), 4-15. doi:10.1111/j.1365-2648.2012.06064.x

Klabunde, C.N., Ambs, A., Keating, N.L., He, Y., Doucette, W.R., Tisnado, D., ... Kahn, K.L. (2009). The role of primary care physicians in cancer care. Journal of General Internal Medicine, 24(9), 1029-1036. doi:10.1007/s11606-009-1058-x

Koch, T. (2006). Establishing rigour in qualitative research: The decision trail. 1993. Journal of Advanced Nursing, 53, 91-100; discussion 101-3.

Lambert, S.D., Loiselle, C.G. et Macdonald, M.E. (2009). An in-depth exploration of information-seeking behavior among individuals with cancer: Part 1 Understanding differential patterns of active information-seeking. Cancer Nursing, 32, 11-25.

Lee, L., Cheung, W.Y., Atkinson, E. et Krzyzanowska, M.K. (2011). Impact of comorbidity on chemotherapy use and outcomes in solid tumors: A systematic review. Journal of Clinical Oncology, 29, 106-117.

Lincoln, Y.S. et Guba, E.G. (1985). Naturalistic inquiry. Beverly Hills, Calif., Sage Publications.

Lindenfeld, J. et Kelly, P.A. (2010). Developing a cardiology-oncology clinical practice guideline. Progress in Cardiovascular Diseases, 53, 173-179.

Louwman, W.J., Janssen-Heijnen, M.L.G., Houterman, S., Voogd, A.C., van der Sangen, M.J.C., Nieuwenhuijzen, G.A. P., \& 
Coebergh, J. W. W. (2005). Less extensive treatment and inferior prognosis for breast cancer patient with comorbidity: A populationbased study. European Journal of Cancer, 41, 779-785.

Lusk, J.M. et Fater, K. (2013). A concept analysis of patient-centered care. Nursing Forum, 48(2), 89-98. doi: 0.1111/nuf.12019

Magilvy, J.K. et Thomas, E. (2009). A first qualitative project: Qualitative descriptive design for novice researchers. Journal for Specialists in Pediatric Nursing, 14, 298-300.

Marengoni, A., Angleman, S., Melis, R., Mangialasche, F., Karp, A., Garmen, A., ... Fratiglioni, L. (2011). Aging with multimorbidity: A systematic review of the literature. Ageing Research Reviews, 10, 430-439.

Meyerhardt, J.A., Catalano, P.J., Haller, D.G., Mayer, R.J., Macdonald, J.S., Benson, A.B. 3rd et Fuchs, C.S. (2003). Impact of diabetes mellitus on outcomes in patients with colon cancer. Journal of Clinical Oncology, 21, 433-40.

Noël, P.H., Chris, F.B., Larme, A.C. et Pugh, J.A. (2005). Collaborative care needs and preferences of primary care patients with multimorbidity. Health Expectations, 8, 54-63.

Partenariat canadien contre le cancer (2016a). Rapport de 2016 sur le rendement du système de lutte contre le cancer. Consulté sur http:// www.systemperformance.ca/reports/

Partenariat canadien contre le cancer (2016b). Nous constatons des progrès : Stratégie canadienne de lutte contre le cancer 2017-2022. Consulté sur http://www.partnershipagainstcancer.ca/wp-content/ uploads/sites/5/2016/02/canadian-strategy-cancer-control-20172022-fr.pdf

Polit, D.F. et Beck, C.T. (2010). Essentials of nursing research: Appraising evidence for nursing practice. Lippincott Williams et Wilkins.

Puts, M.T.E., Papoutsis, A., Springall, E. et Tourangeau, A.E. (2012). A systematic review of unmet needs of newly diagnosed older cancer patients undergoing active cancer treatment. Supportive Care in Cancer, 20, 1377-1394.

Ritchie, C.S., Kvale, E. et Fisch, M.J. (2011). Multimorbidity: An issue of growing importance for oncologists. Journal of Oncology Practice, 7(6), 371-374.

Rodgers, B.L. et Cowles, K.V. (1993). The qualitative research audit trail: A complex collection of documentation. Research in Nursing et Health, 16, 219-226.

Saultz, J.W. et Albedaiwi, W. (2004). Interpersonal continuity of care and patient satisfaction: A critical review. The Annals of Family Medicine, 2, 445-451.
Sitzia, J. et Wood, N. (1998). Study of patient satisfaction with chemotherapy nursing care. European Journal of Oncology Nursing, 2, 142-153.

Smith, A.W., Reeve, B.B., Bellizzi, K.M., Harlan, L.C., Klabunde, C.N., Amsellem, M., ... Hays, R.D. (2008). Cancer, comorbidities, and health-related quality of life of older adults. Health Care Finance Review, 29, 41-56.

Société canadienne du cancer (2012). Vue d'ensemble des statistiques sur le cancer. Consulté sur http://www.cancer.ca/fr-ca/ cancer-information/cancer-101/cancer-statistics-at-a-glance

Société canadienne du cancer, Comité consultatif des statistiques canadiennes sur le cancer (2015). Statistiques canadiennes sur le cancer 2015. Consulté sur http://www.cancer.ca/ /media/cancer.ca/CW/ cancer\%20information/cancer\%20101/Canadian\%20cancer\%20 statistics/Canadian-Cancer-Statistics-2015-FR.pdf?la=fr-CA

Srokowski, T.P., Fang, S., Hortobagyi, G.N. et Giordano, S.H. (2009). Impact of diabetes mellitus on complications and outcomes of adjuvant chemotherapy in older patients with breast cancer. Journal of Clinical Oncology, 27, 2170-2176.

Swanson, J. et Koch, L. (2010). The role of the oncology nurse navigator in distress management of adult inpatients with cancer: A retrospective study. Oncology Nursing Forum, 37, 69-76.

Thompson, A. (2007). The meaning of patient involvement and participation in health care consultations: A taxonomy. Social Science et Medicine, 64, 1297-1310.

Tinetti, M.E., Bogardus, S.T. Jr. et Agostini, J.V. (2004). Potential pitfalls of disease-specific guidelines for patients with multiple conditions. The New England Journal of Medicine, 351, 2870-2874.

Tuckett, A.G. (2005). Part II. Rigour in qualitative research: complexities and solutions. Nurse Researcher, 13, 29-42.

van Der, M. 1999. Relating information needs to the cancer experience: 1. Information as a key coping strategy. European Journal of Cancer Care, 8, 238-244.

van den Akker, M., Buntinx, F. et Knottnerus. (196). Comorbidity or multimorbidity. European Journal of Clinical Practice, 2, 65-70.

Wolf, I., Sadetzki, S., Catane, R., Karasik, A. et Kaufman, B. (2005). Diabetes mellitus and breast cancer. The Lancet Oncology, 6, 103-111.

Yancik, R., Wesley, M.N., Ries, L.A., Havlik, R.J., Long, S., Edwards, B.K. et Yates, J.W. (1998). Comorbidity and age as predictors of risk for early mortality of male and female colon carcinoma patients: A population-based study. Cancer, 82, 2123-34. 